\title{
Analysing Acceleration for Motion Analysis
}

\author{
Yan Sun
}

Jonathon S. Hare

Mark S. Nixon

\author{
Electronics and Computer Science \\ University of Southampton \\ Southampton, UK \\ \{ys1d14,jsh2, msn\}@ecs.soton.ac.uk
}

\begin{abstract}
Previous research in motion analysis of image sequences has generally not considered the basic nature of higher orders of motion such as acceleration. In this work, we disambiguate different types of motion, and in particular focus on acceleration. First, we show acceleration can be computed in a principled manner by extending Horn and Schunck's algorithm for global optical flow estimation. We then demonstrate an approximation of the acceleration field using an alternative established optical flow technique, since most real motions violate the global smoothness assumption of Horn and Schunck. Furthermore, we decompose acceleration into radial and tangential components for greater depth of understanding of the motion. As a general motion descriptor, we show how acceleration provides the capability for differentiating different types of motion in video sequences.
\end{abstract}

Keywords—optical flow; acceleration; motion classification;

\section{INTRODUCTION}

An image is a snapshot in which all motions are frozen in time. This implies that video involves many motions which coalesce to form the image sequence. Nowadays, computer vision approaches can differentiate objects in motion from those which are static, but little more [1], [2]. In actuality, there are many different types of motion: in the simplest sense, there are objects that move with velocity and some that move with acceleration; many objects have more complicated motions. This paper describes a new approach designed to disambiguate different types of motion starting with the detection of objects moving with acceleration via established computer vision techniques.
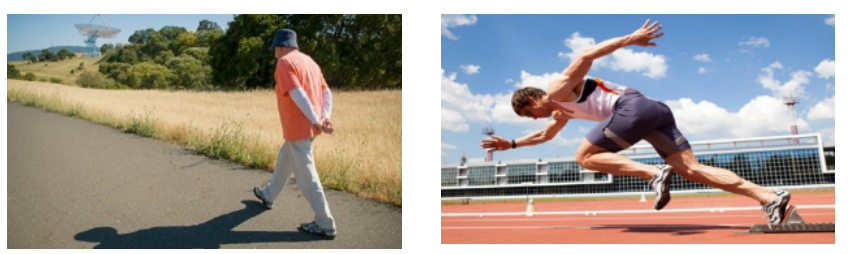

Fig. 1. Different types of motion.

(from: http://news.stanford.edu/ and http://www.wisegeek.com/)

Fig. 1 illustrates the diversity of motion, the man in the left image is walking with constant velocity in general and the athlete on the right is speeding up, or accelerating. Each part of both men is experiencing different types of motion, especially their legs. Consider a walking person: the body is approximately at a constant velocity, and one of the legs is stationary to support the body while the other one is swinging forward like a pendulum, as shown in Fig. 2. These motions can be identified by acceleration because once the status of an object has changed, there must be acceleration. Therefore, we hypothesise that we can find the legs of a person's body and discriminate between them by extracting their acceleration features.

Apart from identifying behaviours, acceleration also offers an alternative approach to understand image content. For examples in autonomous driving, the acceleration of other cars can help a computer to make correct decisions about other vehicles' motion trajectories.

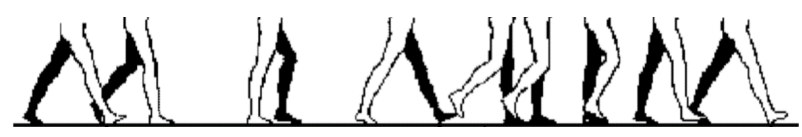

Fig. 2. A walking cycle [12].

Beyond our previous work [3], the new contributions are summarized below:

- We extend the original Horn Schunck method optical flow technique to focus on acceleration.

- Our analysis shows the constraints to be too stringent for application in real-world video footage, so we then explore the use of the other state of art optical flow algorithms as basis approximating acceleration with wider applicability in general video.

- We show generalised application of acceleration detection in a richer selection of imagery for not only the new approach but also the new basis, on synthetic and on real image sequences.

- We compared the capability of discriminating motion characteristics between velocity and acceleration flow and show the advantages of acceleration.

This paper is arranged as follows: Section 2 presents the algorithm for estimating acceleration from optical flow and shows experimental results both on synthetic and real-world image sequences. Section 3 shows how acceleration can be decomposed into radial and tangential components as well as experimental results, and demonstrates the ability of acceleration that disambiguate different types of motion. Section 4 discusses and provides conclusions of this work. 


\section{ESTIMATION OF ACCELERATION FLOW}

There has been little work as yet analysing acceleration before we determined gait events through acceleration flow in our earlier work [3]. Beyond that, [4] differentiated the velocity field without considering an analytic solution for isolating acceleration whereas [5] extended the brightness constraint to three frames and solved the problem in an extremely complex manner. Our own previous work [3] did not include the following differential basis and was focused more on the analysis of human gait detecting in particular the heel strikes. Our new approach in this paper retains the elegance of the original Horn Schunck formulation with an approach that isolates only acceleration, thus allowing more detailed analysis of complex motion fields, and we provide a more general experimental analysis.

\section{A. Recovering Acceleration Flow from Optical Flow}

Our new algorithm is inspired by Horn and Schunck's work [6], the earliest variational approach for recovering optical flow. If $\mathbf{I}(x, y, t)$ denotes the image intensity on $(x, y)$ at time $t$, we extend the initial hypothesis that the image intensity is constant during three frames:

$$
\begin{aligned}
\mathbf{I}\left(x-\delta x_{1}, y-\delta y_{2}, t-\delta t\right) & =\mathbf{I}(x, y, t) \\
& =\mathbf{I}\left(x+\delta x_{2}, y+\delta y_{2}, t+\delta t\right)
\end{aligned}
$$

Expanding (1) by Taylor expansion:

$$
\begin{aligned}
\mathbf{I}_{x}\left(-\delta x_{1}\right)+\mathbf{I}_{y}\left(-\delta y_{1}\right)+\mathbf{I}_{t} & (-\delta t) \\
& =\mathbf{I}_{x} \delta x_{2}+\mathbf{I}_{y} \delta y_{2}+\mathbf{I}_{t} \delta t
\end{aligned}
$$

where the higher order terms are ignored. Dividing (2) by $\delta t$, the gradient constraint is yielded:

$$
\nabla \mathbf{I} \cdot \mathbf{v}_{t-\delta t}+\mathbf{I}_{t-\delta t}=\nabla \mathbf{I} \cdot \mathbf{v}_{t+\delta t}+\mathbf{I}_{t+\delta t}
$$

$$
\nabla \mathbf{I}\left(\mathbf{v}_{t}-\mathbf{a} \delta t\right)+\mathbf{I}_{t-\delta t}=\nabla \mathbf{I}\left(\mathbf{v}_{t}+\mathbf{a} \delta t\right)+\mathbf{I}_{t+\delta t}
$$

where $\mathbf{v}_{t}$ denotes the velocity vector at time $t$ and the acceleration vector $\mathbf{a}$ is composed by horizontal and vertical components $\left(a_{u}, a_{v}\right)^{T}$. Differentiating (4) with respect to time, we can obtain the Optical Flow Constraint Equation (OFCE) of acceleration:

$$
\nabla \mathbf{I} \cdot 2 \mathbf{a}+\mathbf{I}_{t t}=0
$$

where $\mathbf{I}_{t t}$ indicates the second order of image intensity respect time.

Acceleration also has similar smoothness characteristics to velocity in that neighbouring pixels tend to have similar acceleration. This shows a natural linkage between velocity and acceleration analysis in image sequences. By following a similar solution to Horn Schunck, we can determine the acceleration flow in images. We now have the basis for detecting acceleration, we shall now move to evaluating this approach to determine whether we can indeed detect acceleration from image intensity.

\section{B. Analysing Synthetic Image Sequences}

We evaluate our new approach first on synthetic images to assess performance before analysis on real images to show application capability. The advantage of synthetic images is that the input signal is without noise, specularity, or other types of noise. Also, the motion field and scene properties can be manipulated as required. This image sequence involving linear motion is synthesized by using images from the Middlebury database [7]. A subpart of a frame from Mequon (the block of two faces in Fig. 3 (a)) in Middlebury is embedded in a frame from the Wooden images. The Mequon sub-frame shifts along a linear trajectory to the lower right corner at speed $1 / 4$ pixel/frame, both on horizontal and vertical axes. Sub-pixel motion is achieved by down-sampling the images.

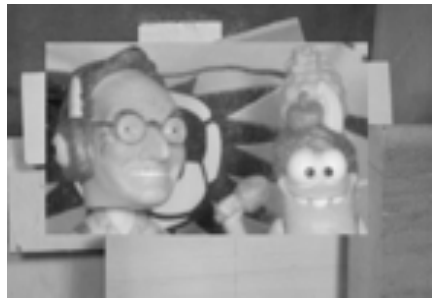

(a) Synthetic image

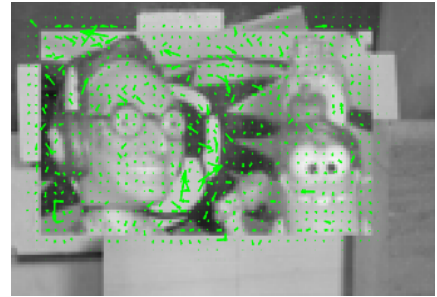

(b) Acceleration field when Mequon is undergoing non-acceleration motion.

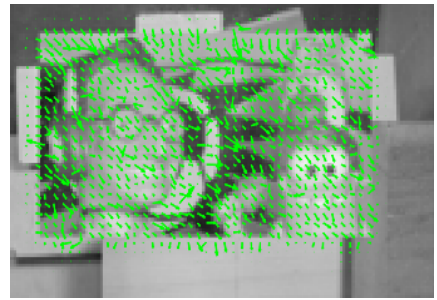

(c) Acceleration field when Mequon is accelerating.

Fig. 3. The detection results of synthetic images.

where $\nabla=\left(\frac{\partial}{\partial x}, \frac{\partial}{\partial y}\right)$, and $\mathbf{v}$ consists of horizontal and vertical components $(u, v)^{T}$.

If the acceleration is dynamic from frame to frame, then this become an ill-posed problem. More commonly, motion is smooth which means acceleration is usually constant during a small period. Here, we assume that the acceleration does not change during three consecutive frames. Then velocity can be presented by $v_{0}+a \delta t$ from Newton's laws:
Fig. 3. (a) shows one example frame of the synthetic sequence, (b) and (c) shows the acceleration detection results under the motion without and with acceleration respectively. When Mequon moves without acceleration, there is little acceleration flow detected, except random noise. Encouragingly, as opposed to the uniform motion the new algorithm detected evenly distributed acceleration flow in the right hand frame. We would have liked to compare performance with the other acceleration technique [5] but the implementation 


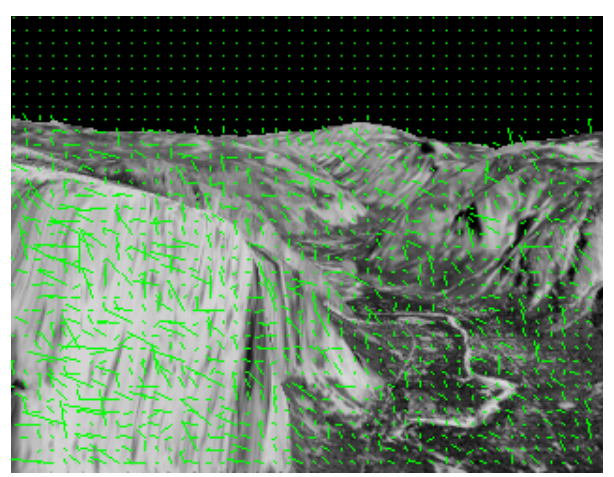

Fig. 4. The experimental result on Yosemite.

is unavailable for that complex technique whereas [4] lacks the analytical basis here.

We also evaluated our new algorithm on the Yosemite image sequence which is a challenge for fundamental optical flow algorithms since the velocities of different areas vary and the edges are occluded between the mountains. The upper right corner translates to the right with a speed of 2 pixels/frame and the speed in the lower left area is about 4 to 5 pixels/frame [8]. The non-uniform motion is caused by the asymmetrical projection of 3-D motion onto the 2-D image surface. Our acceleration measurement produces a poor result as shown in Fig. 4 since Horn and Schunck's algorithm assumes global smoothness and sub-pixel motion which is violated in this case.

For more precise, TABLE I reports the performance measures of our acceleration estimation algorithm on Middlebury optical flow benchmark data. We computed the average Angular Error (AE) and Standard Deviation (SD) between the experimental results and pseudo ground truth. Since the ground truth flow can be accessed from the Middlebury optical flow database is only between two frames in the dataset, we use the acceleration flow estimated based on MDP-Flow2 [9] by (6) as the pseudo ground truth. MDP-Flow2 is a highly-rated optical flow estimation algorithm on the Middleburry evaluation website $^{1}$. The results in Table I show that the new acceleration estimation algorithm is not accurate enough to be used as a motion descriptor because of the stringent constraints. Fig. 5. shows the colour map of the pseudo ground truth and estimated acceleration flow of data "Backyard" and "Walking". In the pseudo ground truth, the falling ball in (c) and the walking legs in (e) has more acceleration than other motion areas. The acceleration field illustrates detection of features consistent with acceleration features, which velocity analysis lacks.

TABLE I. Error analysis of acceleration estimation algorithm

\begin{tabular}{|c|c|c|c|c|c|c|c|c|}
\hline DE & Army & Mequon & Schefflera & $\begin{array}{c}\text { Backy } \\
\text { ard }\end{array}$ & $\begin{array}{c}\text { Dumptr } \\
\text { uck }\end{array}$ & $\begin{array}{c}\text { Grove2 } \\
\text { RubberW } \\
\text { hale }\end{array}$ & Walking \\
\hline Average $\left(^{\circ}\right)$ & 10.84 & 28.3 & 33.89 & 17.82 & 12.36 & 14.31 & 9.74 & 17.82 \\
\hline SD $\left(^{\circ}\right)$ & 8.61 & 20.18 & 19.77 & 21.59 & 17.98 & 13.82 & 8.77 & 19.54 \\
\hline
\end{tabular}

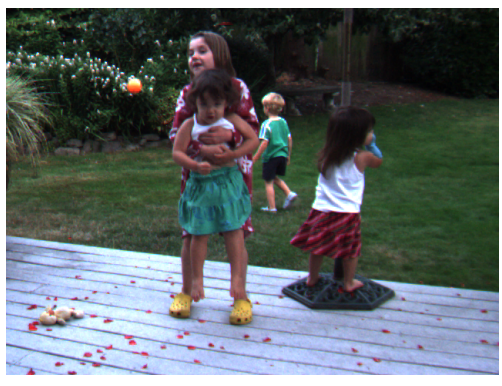

(a) Backyard

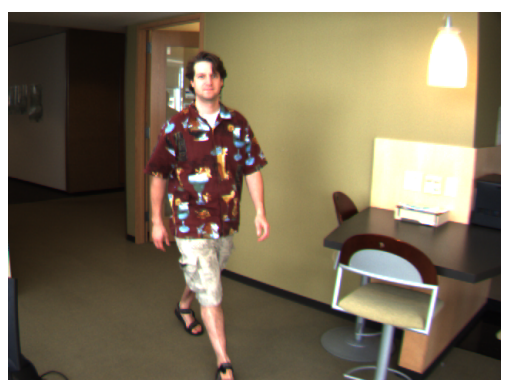

(d) Walking

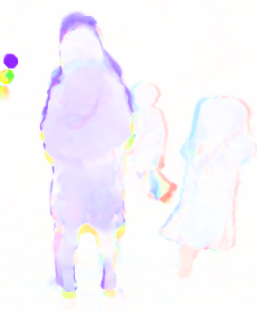

(b) Backyard pseudo ground truth

(c) Backyard acceleration

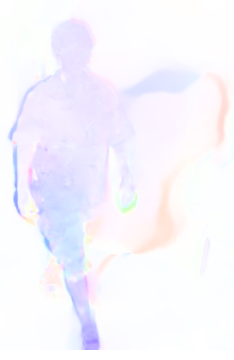

(e) Walking pseudo ground truth
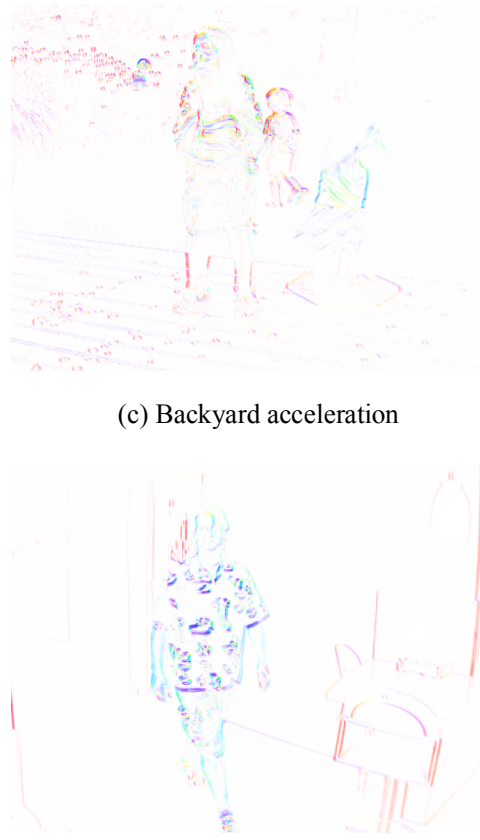

(f) Walking acceleration

Fig. 5. The colour map of acceleration flow: (b), (e) are pseudo ground truth [7] and (c), (f) detection by the new approach on the right.

\footnotetext{
${ }^{1}$ http://vision.middlebury.edu/flow/eval/
} 


\section{Estimating Acceleration via Other Flow Estimation Methods}

Since the motion in real images is often large we want to seek more generalized form for recovering acceleration from image sequences, we opt to use the state of the art optical flow estimation algorithm to approximate the acceleration than to use the new algorithm. In this paper, we use DeepFlow [10], which is a popular new technique with excellent performance for large displacement estimation and non-rigid matching as our fundamental technique of our algorithm.

As in our previous work [3], we approximate the acceleration by differencing the velocity field between frames. By reference to the same starting position, the time axis is reversed when estimating the previous velocity $\mathbf{v}_{t}$ :

$$
\mathbf{a}_{t}=\mathbf{v}_{t+1}-\left(-\mathbf{v}_{t}\right)
$$

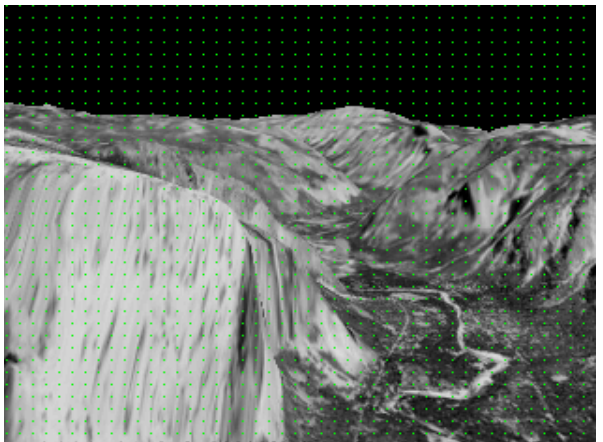

(a) Moving with constant velocity. motion field, which can be found in [7]. We now have the basis for detecting acceleration and its extension to a more generalized form that able to detect acceleration under large motions. In real image sequences, detecting acceleration flow can help distinguish objects undergoing different motions. In Fig. 7, the silver car in the front and the red dump truck in the back are approximately moving in constant velocity. As contrast, the detection result shows the other two cars are accelerating.

\section{TANGENTIAL AND RADIAL ACCELERATION}

Acceleration for motion is composed of two components: tangential and radial acceleration. The tangential component changes the magnitude of the velocity vector and the direction is located in the tangent line of the trajectory (increasing or decreasing the speed). The radial component (also called centripetal acceleration in circular motion) changes the direction of the velocity and it points to the centre of the curved path

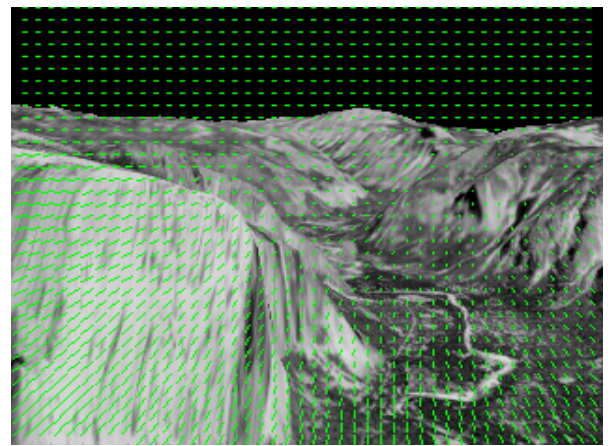

(b) Moving with acceleration.

Fig. 6. The experiment results on Yosemite.

The new results in Fig. 6. show this more general approach when used to estimate acceleration flow. In (a), the scene is moving with constant velocity so there is little acceleration flow. In (b), the pixels in the lower left corner have larger acceleration than the upper right corner and the whole scene is accelerating to the upper left corner, which shows the consistency with the known motion characteristics of the Yosemite sequence. This is a considerable improvement over Fig. 4 which used the Horn Schunck formulation.

Since our algorithm is based on an established optical flow detection technique, the performance of acceleration estimation depends on the performance of DeepFlow for estimating the

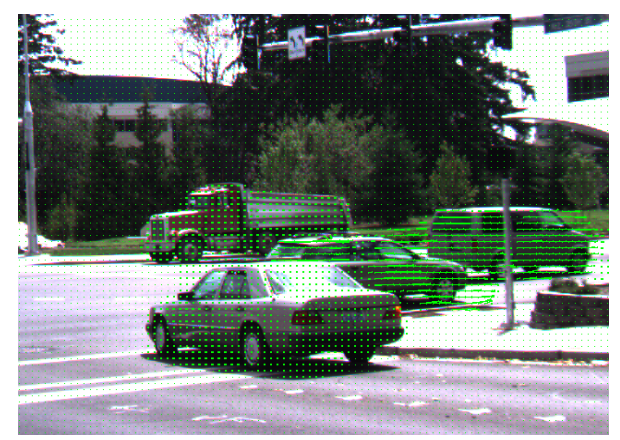

Fig. 7. Detecting acceleration on real images [7]. (normal to the direction of velocity), as shown in Fig. 8. Motion is composed of linear or circular motion; therefore, the motion incorporated in images is either linear or circular if the time interval is sufficiently small.

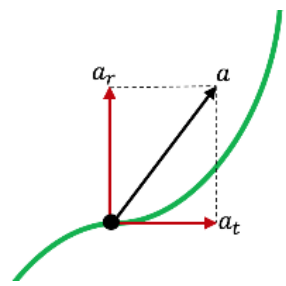

Fig. 8. The acceleration of a particle along arbitrary curved path is composed of radial and tangential component.

\section{A. Decomposing Tangential and Radial Components from Acceleration}

We assume that the moving points which follow the curved trajectories rotate along the same arc in any three consecutive frames since three nonlinear points can determine one and only one circle. The rotation centre can be calculated by the positions of the pixel in the consecutive frames. Connecting these three points with straight lines and applying perpendicular bisectors to them, the centre of the circle is then located at the intersection of the two perpendicular bisectors. 


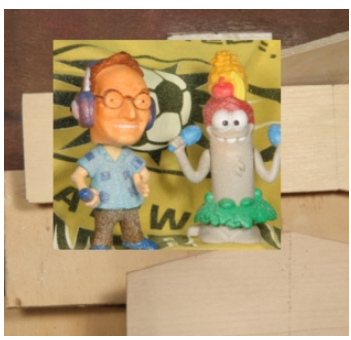

(a) $\delta x=0, \delta y=0$

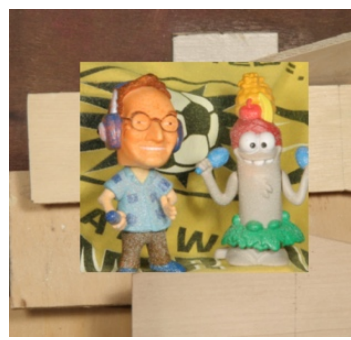

(b) $\delta x=32, \delta y=32$

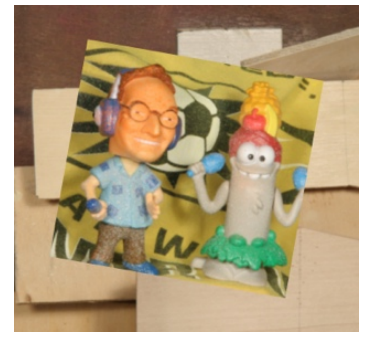

(c) $\delta \alpha=10^{\circ}$

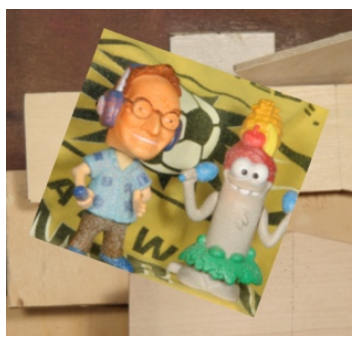

(d) $\delta \alpha=30^{\circ}$

Fig. 9. Examples of artificial motion.

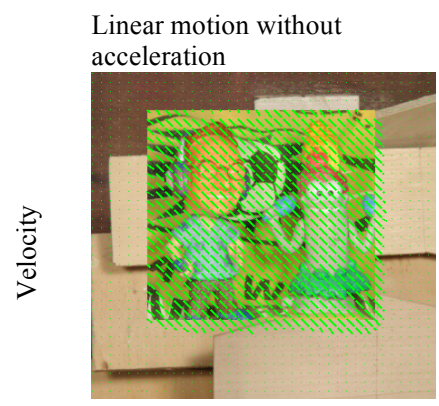

Linear motion with

acceleration
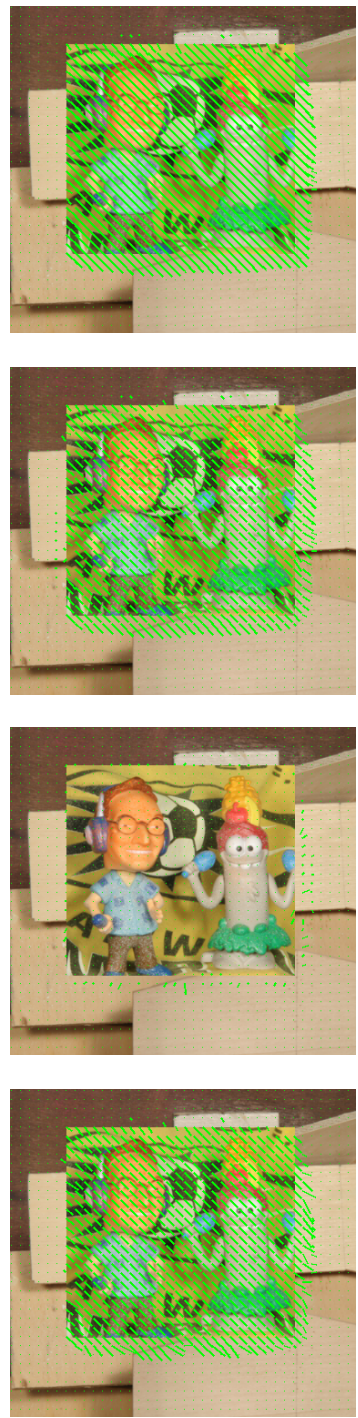

Circular motion without

angular acceleration
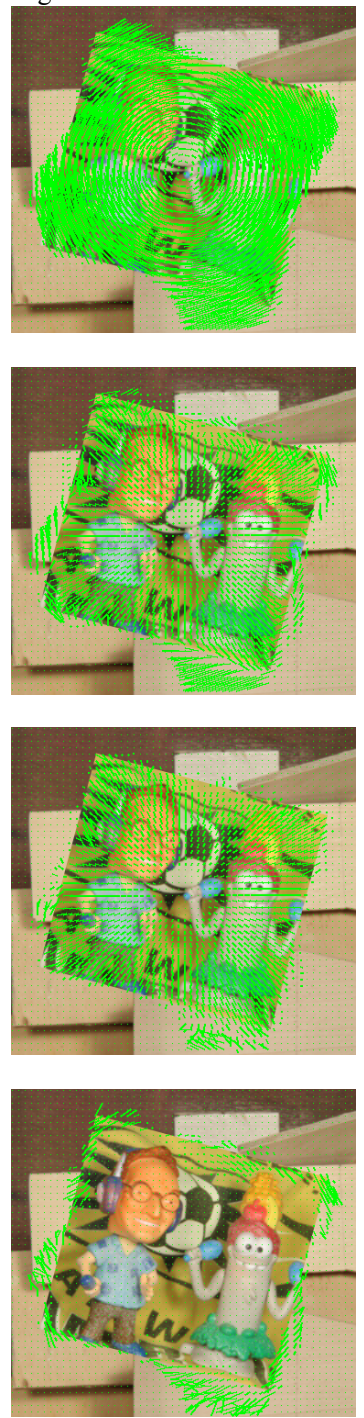

Circular motion with angular acceleration
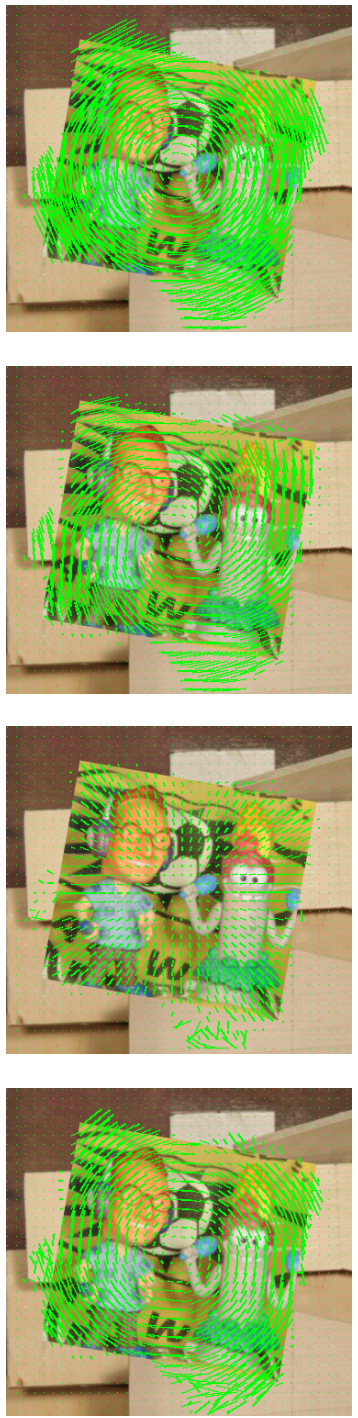

Fig. 10. The experimental results of synthetic images.

Suppose the coordinates of a point in three consecutive frames are: $\mathbf{p}_{i}\left(x_{i}, y_{i}\right) i \in(t-1, t, t+1)$, mo, no are the perpendicular bisectors of $\mathbf{p}_{t-1} \mathbf{p}_{t}$ and $\mathbf{p}_{t} \mathbf{p}_{t+1}$, and $\mathbf{o}\left(x_{o}, y_{o}\right)$ denotes the center of the circular motion, hence:

$$
\overrightarrow{\mathbf{m o}} \cdot \overrightarrow{\mathbf{p}_{t-1} \mathbf{p}_{t}}=\overrightarrow{\mathbf{n o}} \cdot \overrightarrow{\mathbf{p}_{t} \mathbf{p}_{t+1}}
$$

then the coordinate of $\mathbf{o}$ can be obtained by: 


$$
\boldsymbol{o}^{T}=0.5 \cdot \boldsymbol{\Phi}^{-1} \boldsymbol{\Psi}
$$

where,

$$
\boldsymbol{\Phi}=\left[\begin{array}{ll}
x_{t}-x_{t-1} & y_{t}-y_{t-1} \\
x_{t+1}-x_{t} & y_{t+1}-y_{t}
\end{array}\right] \quad \boldsymbol{\Psi}=\left[\begin{array}{c}
x_{t}{ }^{2}-x_{t-1}{ }^{2}+y_{t}{ }^{2}-y_{t-1}{ }^{2} \\
x_{t+1}{ }^{2}-x_{t}{ }^{2}+y_{t+1}{ }^{2}-y_{t}{ }^{2}
\end{array}\right]
$$

We use $a=\left(x_{t}+a_{u}, y_{t}+a_{v}\right)$ to present the coordinates of acceleration vector in image plane. The positions of tangential acceleration $\tan (u, v)$ and radial acceleration $\operatorname{rad}(u, v)$ can be estimated by:

$$
\begin{aligned}
& \boldsymbol{t a n}^{T}=\left[\begin{array}{lll}
\mathbf{f}(-\theta) & \mathbf{g}(-\theta)
\end{array}\right]^{T}\left[\mathbf{p}_{t} \cdot \mathbf{f}(\theta) \quad \mathbf{a} \cdot \mathbf{g}(\theta)\right]^{T} \\
& \boldsymbol{r a d}^{T}=\left[\begin{array}{lll}
\mathbf{f}(-\theta) & \mathbf{g}(-\theta)
\end{array}\right]^{T}\left[\begin{array}{ll}
\mathbf{a} \cdot \mathbf{f}(\theta) & \mathbf{p}_{t} \cdot \mathbf{g}(\theta)
\end{array}\right]^{T}
\end{aligned}
$$

where $\theta$ is the angle between $\mathbf{o p}_{t}$ and the horizontal axis $\mathbf{f}(\theta)=(\cos \theta, \sin \theta), \mathbf{g}(\theta)=(-\sin \theta, \cos \theta)$.

We now have the basis for detecting acceleration and its extension to a more generalized form. In the next section, we evaluate these approaches to determine whether we can indeed detect acceleration from image sequences.

\section{B. Deploying Decomposed Algorithm on Synthetic Image Sequences}

We evaluate the decomposed approach first on synthetic images to assess performance before analysis on real images to show capability in real applications. We manipulate Mequon sequence by rotating around its centre in the synthetic sequence to form circular motion. Examples of test image are illustrated in Fig 10.

The experiments are classified into 4 groups: linear shift with constant velocity, linear shift with acceleration, rotation in constant angular velocity and rotation with angular acceleration. We detected velocity, resultant acceleration, radial and tangential fields for each group separately and illustrate the results in Fig. 10.

In the example of linear shift, there is little radial acceleration because the direction of trajectory does not change and the tangential component only appears when the object is accelerating. The resultant acceleration field shows similar features with tangential acceleration since it only contains the
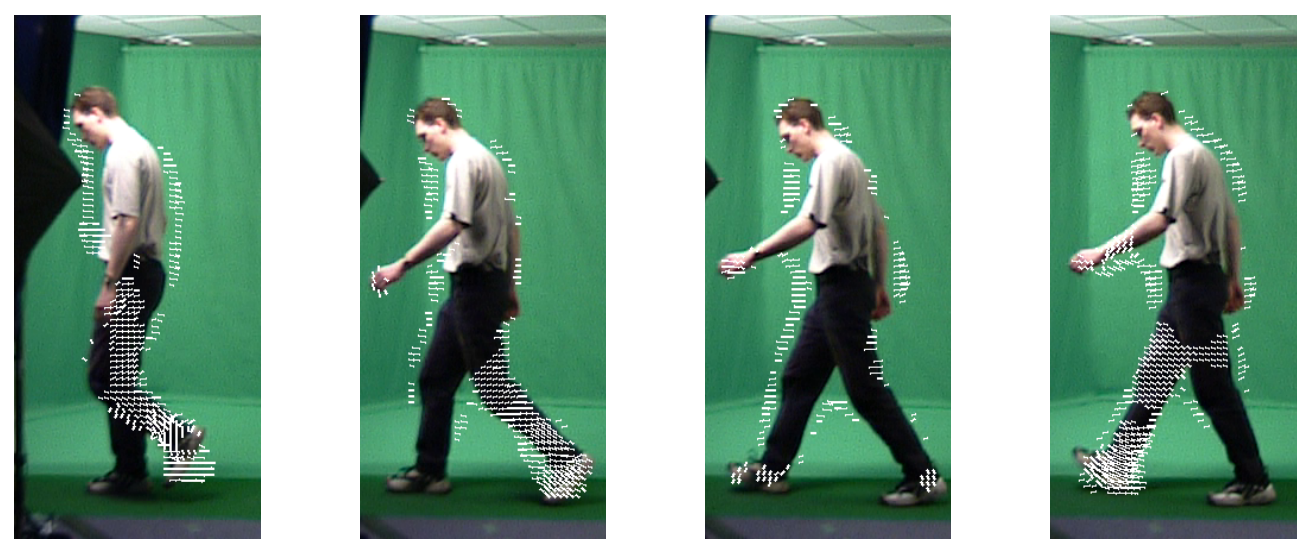

(a) Acceleration flow on a walking person.
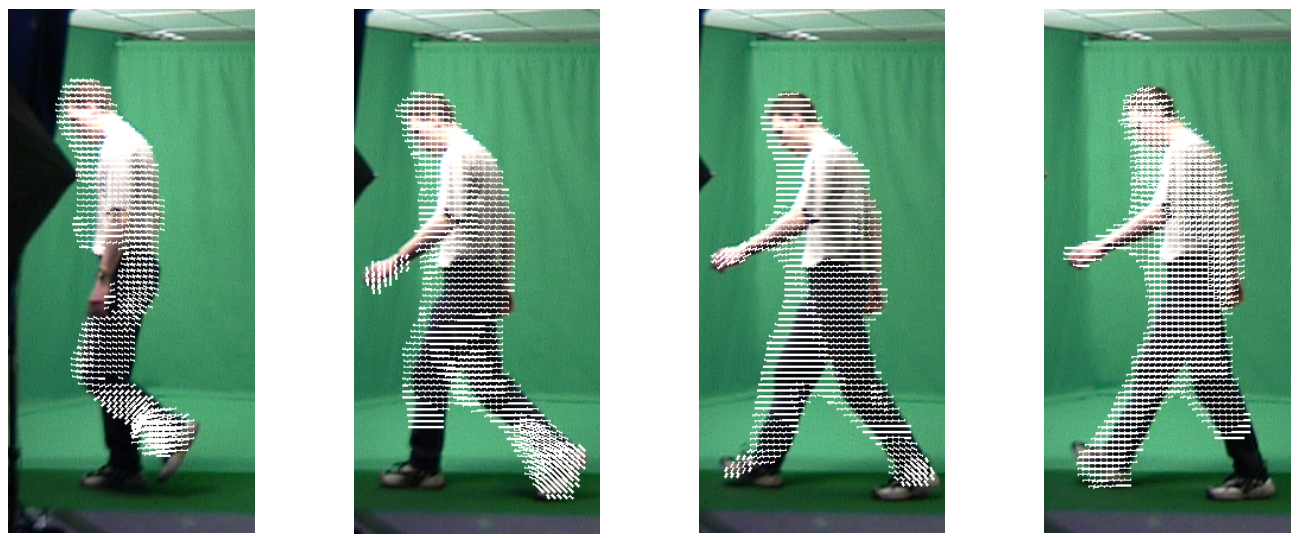

(b) Velocity flow on a walking.

Fig. 11. Analysing acceleration and velocity flow on real images.
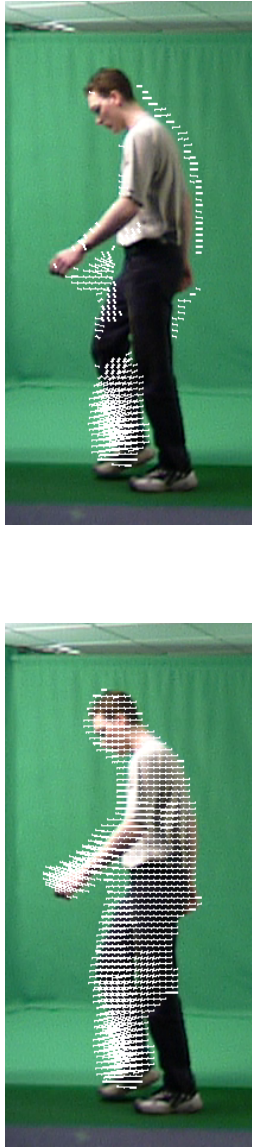
tangential component in linear displacement. Velocity appears in both situations however.

In the rotation examples, radial acceleration appears both under rotation with constant angular velocity, and under angular acceleration, due to the direction of motion changing all the time. The magnitude of radial acceleration is increasing with the angle of the object rotated. All the radial acceleration field points to the Mequon sub-frame centre since the sub-frame rotates about it. The directions of tangential components are along the tangent of rotating trajectory showing a result consistent with expectations. Simultaneously, the velocity field does not show any obvious distinction. There appears to be some noise around the edge of the moving object; this is mainly caused by discontinuous motion in that area. Our estimated detection of acceleration shows expected results on artificial scenes. We have used our new technique to detect heel strike for gait analysis in our previous work [3].

\section{Deploying the Algorithm on Real Images}

We also provide a new example of our approach detecting acceleration on images of a subject walking in a chroma key laboratory [11], in Fig. 11. Acceleration is detected mainly only around the limbs of walking subject and is maximum around the leg which is swinging forward when the other leg is in the stance phase, since the limbs appear to have pendulum-like motion when people are walking [12]. We also detect the outer parts of the upper thorax, which is consistent with its inverted pendulum motion. In contrast, the velocity flow is distributed all over the body without notable difference, so the detected acceleration is consistent with the above analysis. Comparison was not possible with [5] due to lack of implementation and [4] used Horn Schunck violating primary assumption as in our new algorithm.

\section{DISCUSSION AND CONCLUSION}

Previous research in motion analysis mostly focused on the displacement between frames, without considering the diversity of motion. Acceleration is a more distinct feature than displacement. Presenting motion by acceleration can help for better understanding the scene. There has been little study on revealing acceleration from optical flow however they lack an analytic solution. In this paper, we derive acceleration from the basis of Horn Schunck but we found most real motion violates the basic global smoothness assumptions made in the Horn Schunck formulation. We show another way to approximate an acceleration field which is more accurate and able to handle most situations, and appears improved over the Horn Schunck technique on the standard Yosemite test sequence. The acceleration is decomposed into constituent parts allow greater depth in the understanding of the motion. The experiments on a variety of image sequences illustrate the ability of acceleration that discriminate different motion whereas velocity did not show any obvious difference. Clearly, acceleration is likely to be more sensitive to noise though the experiments show that this is not a sever limitation and in fact radial acceleration error estimates are encouragingly low. The ability of the new technique is also demonstrated by its capability to achieve radial and tangential acceleration analysis, providing a completely new way to understand and disambiguate motions in image sequences.

\section{REFERENCES}

[1] D. Sun, S. Roth, and M. J. Black, “A quantitative analysis of current practices in optical flow estimation and the principles behind them," Int. J. Comput. Vis., vol. 106, no. 2, pp. 115-137, 2014.

[2] D. Fortun, P. Bouthemy, and C. Kervrann, "Optical flow modeling and computation: A survey,” Comput. Vis. Image Underst., vol. 134, pp. 1-21, 2015 .

[3] Y. Sun, J. S. Hare, and M. S. Nixon, "Detecting Acceleration for Gait and Crime Scene Analysis," in International Conference on Imaging for Crime Detection and Prevention, 2016.

[4] Z. Dong, J. Qin, and Y. Wang, "Multi-stream deep networks for person to person violence detection in videos," in Communications in Computer and Information Science, 2016, vol. 662, pp. 517-531.

[5] C. Chen, Y. Shao, and X. Bi, "Detection of Anomalous Crowd Behavior Based on the Acceleration Feature,” IEEE Sens. J., vol. 15, no. 12, pp. 7252-7261, 2015.

[6] B. K. B. Horn and B. G. Schunck, "Determining Optical Flow," Artif. Intell., vol. 17, no. 1-3, pp. 185-203, 1981.

[7] S. Baker, D. Scharstein, J. P. Lewis, S. Roth, M. J. Black, and R. Szeliski, "A database and evaluation methodology for optical flow," Int. J. Comput. Vis., vol. 92, no. 1, pp. 1-31, 2011.

[8] J. L. Barron, D. J. Fleet, and S. S. Beauchemin, "Performance of optical flow techniques,” Int. J. Comput. Vis., vol. 12, no. 1, pp. 4377, Feb. 1994.

[9] L. Xu, J. Jia, and Y. Matsushita, "Motion detail preserving optical flow estimation," IEEE Transactions on Pattern Analysis and Machine Intelligence, vol. 34, no. 9, pp. 1744-1757, 2012.

[10] P. Weinzaepfel, J. Revaud, Z. Harchaoui, and C. Schmid, "DeepFlow: Large displacement optical flow with deep matching," in Proceedings of the IEEE International Conference on Computer Vision, 2013, pp. 1385-1392.

[11] J. Shutler, M. Grant, M. S. Nixon, and J. N. Carter, "On a Large Sequence-Based Human Gait Database," in Proceedings Fourth International Conference Recent Advances in Soft Computing, Berlin, Heidelberg: Springer Berlin Heidelberg, 2002, pp. 66-72.

[12] D. Cunado, M. S. Nixon, and J. N. Carter, "Automatic extraction and description of human gait models for recognition purposes," Comput. Vis. Image Underst., vol. 90, no. 1, pp. 1-41, 2003. 